\title{
Expression of genetic and environmental variation for life history characters on the usual and novel hosts in Callosobruchus maculatus (Coleoptera: Bruchidae)
}

\author{
TADEUSZ J. KAWECKI* \\ Institute of Zoology, University of Basel, Rheinsprung 9, CH-4051 Basel, Switzerland
}

\begin{abstract}
Elevated levels of genetic and environmental variance are often expressed in extreme habitats, but it is unclear whether it is because of their novelty, or their low quality. Using a half-sib-full-sib design, I measured the components of phenotypic variance in life-history traits (developmental time, emergence weight, growth rate, lifetime fecundity and male life span), and heritability of larval survival, expressed by the cowpea weevil, Callosobruchus maculatus, on the usual and three novel host species. Because the novel hosts differed in quality, I was to some extent able to distinguish between the effects of host novelty and quality. For all traits except larval survival the among-sire variance component was consistently higher on the usual host, although this result was statistically significant only when the estimates for different traits were standardized and pooled. It contradicts the predictions of evolutionary theories. In turn the dam component for these traits tended to be smallest on the usual host, but this pattern was less pronounced. The within-family variance component decreased with improving performance. This result supports the hypothesis that canalization of development against environmental fluctuations is less efficient in poor habitats. I found no effects of host novelty on environmental variance. Heritability of larval survival was lowest on the usual host, in accord with the hypothesis that predicted higher expression of genetic variance in novel environments.
\end{abstract}

Keywords: habitat variation, heritability, heterogeneous environments, life history, seed beetles, variance components.

\section{Introduction}

The amount of genetic variation in fitness components expressed in a novel environment crucially affects the chances of evolving adaptations to that environment and thus broadening the ecological niche. Two alternative predictions for the effects of novel environments on genetic variation exist. On the one hand, many generations of natural selection in the usual environment should have depleted genetic variation in fitness traits expressed in that environment (Robertson, 1955), whereas at least a part of genetic variation in fitness components expressed in the new environment should have been neutral. More genetic variation should therefore be expressed in the novel environment. This argument requires that fitness in these two habitats is in part affected by different sets of genes. On

*Correspondence: Department of Zoology, Division of Ecology, University of Helsinki, PO Box 17, SF-00014 Helsinki, Finland the other hand, the effects of many alleles may be compensated for in an optimal environment, and thus have little impact on fitness components (genetic canalization; Waddington, 1940; Stearns \& Kawecki, 1995). Under harsh, stressful conditions the compensatory mechanisms involved in genetic canalization may fail, resulting in an enhanced expression of allelic variation at the level of life history (reviewed in Hoffmann \& Parsons, 1991). This hypothesis predicts therefore that genetic variation should increase with decreasing quality of the environment.

Analogous hypotheses can be formulated for the environmental variance component. Canalization mechanisms buffering ontogeny against environmental perturbation and developmental noise may be expected to fail under stress. Alternatively, a novel environment might exert new kinds of microenvironmental disturbances, for which no canalizing mechanisms would have evolved. Hence the alternative hypotheses that higher environmental variance should 
be expressed in a novel environment, or in a poor environment.

This paper reports the results of an experiment in which I measured components of phenotypic variance in fitness-related traits expressed by a herbivorous insect, the cowpea weevil, on four host species. One of them (mung bean) had constituted the environment of this population for at least the last 150 generations; others were novel. Although many studies of herbivorous insects have measured the effect of different hosts on life history traits, few have compared patterns of variation in these traits expressed in the usual and novel environments (Holloway et al., 1990; Via, 1991). In contrast to those studies, I used three novel host species with different effects on life history. This offered a possibility to distinguish between the influences of host quality and novelty on variance components, and thus test whether more genetic and environmental variation is expressed in a novel environment (host novelty hypothesis) or in a poor environment (host quality hypothesis).

\section{Materials and methods}

The cowpea weevil, Callosobruchus maculatus (F.) is a cosmopolitan polyphagous pest of stored legumes in the tropics. Its natural environment, grain stores, is easily replicable in the laboratory. Larvae complete development within a single bean; the adults do not feed, so that adult performance is largely determined by the amount of resource accumulated at the larval stage. I used the 'South India' strain of $C$. maculatus, established in 1979 (Thantianga \& Mitchell, 1990), and since then maintained in the laboratory on mung beans, with the population size always above 200 (Messina, 1991; Mitchell, 1991). My cultures of the South India strain originate from more than 300 individuals obtained from Dr Frank Messina in June 1992. I applied a variable culture interval (21-28 days) to reduce selection on developmental time. The South India is an extreme 'contest' strain, where usually only a single adult emerges from a bean, and females avoid ovipositing on beans that already contain eggs (Messina, 1990).

The four host species used in this experiment were mung bean Vigna radiata (the usual host), black gram Vigna mungo, adzuki Vigna angularis, and red bean Phaseolus sp. The population has been maintained on mung bean for more than 150 generations; the remaining three hosts can therefore be viewed as novel. These hosts have strong and complex effects on the life history of this population of cowpea weevil, red bean being a better host than mung (T. J. Kawecki, unpublished data).
The observational components of phenotypic variance were estimated in a classic half-sib-full-sib design (Falconer, 1989), with 64 sires and up to three dams per sire (T. J. Kawecki, unpublished data). Each dam oviposited on all four host species, and up to four offspring per host species per dam were obtained. There was always only one larva per bean (i.e. no competition). I measured posthatching larval survival, developmental time, emergence weight, lifetime fecundity and the life span of unmated males. Posthatching survival was defined as the number of adults divided by the number of hatched eggs; an egg was classified as unhatched if there were no signs of development or if the larva died before entering the seed. Failure of eggs to hatch could result from fertilization failure and including them would inflate heritability estimates. For many life history traits variance is expected to be proportional to the mean by default (Sokal \& Rohlf, 1981). To remove this scaling effect the measurements of the remaining four traits were transformed by taking natural logarithms; this also usually improved normality of the data. Using nontransformed data did not change the conclusions qualitatively. In addition to the five characters above, I estimated variance components for average growth rate, residual fecundity and residual life span. Growth rate was defined as $\ln$ (emergence weight)/ $\ln$ (developmental time). Fecundity and life span increase with body size; residual fecundity and residual male life span were corrected for this effect. They were obtained as residuals from a regression of $\ln$ (fecundity), and of $\ln$ (life span), respectively, on $\ln$ (emergence weight). To obtain the slope of this regression, I pooled all data within hosts, and performed an analysis of covariance, with host as the group variable and emergence weight as the covariate. The slope thus represents the change of fecundity (or life span) with weight within hosts, not among hosts (Sokal \& Rohlf, 1981).

The sample sizes were, depending on host, 675-691 for larval survival; 561-647 for developmental time, emergence weight and growth rate, 266-324 for fecundity and 264-315 for life span.

\section{Estimation of variance components and heritabilities}

I estimated the observational components of variance (among sires $\sigma_{\mathrm{S}}^{2}$, among dams within sires $\sigma_{\mathrm{D}}^{2}$, and among offspring within full-sib families $\sigma_{\mathrm{W}}^{2}$ ) for each trait except for posthatching survival (see below) expressed on each host, using the restricted maximum likelihood approach (procedure VARCOMP, option REML; SAS Institute, 1989). To test whether $\sigma_{\mathrm{S}}^{2}$ and $\sigma_{\mathrm{D}}^{2}$ differed from zero, I ran a parallel series of ANOvAs for each host and trait (procedure GLM, type IV sum of 
squares). Sex was included as a fixed effect in the model for traits measured on both sexes.

Total phenotypic variance $V_{\mathrm{P}}$ was calculated as the sum of the three observational components. The (narrow-sense) heritability was estimated as $h_{\text {Sire }}^{2}=4 \sigma_{\mathrm{S}}^{2} / V_{\mathrm{P}}$ (Falconer, 1989). I also calculated heritability estimates based on the dam component as $h_{\mathrm{Dam}}^{2}=4 \sigma_{\mathrm{D}}^{2} / V_{\mathrm{p}}$. Because of strong maternal effects in the cowpea weevil (Fox, 1993) $h_{\text {Dam }}^{2}$ overestimates the broad-sense heritability (Falconer, 1989). The ANOVAs testing for significance of $\sigma_{\mathrm{S}}^{2}$ and $\sigma_{\mathrm{D}}^{2}$ (above) provided the significance of narrow-sense and broad-sense heritability estimates. The standard errors of the variance components were estimated with the jackknife technique (Arvesen \& Schmitz, 1970; Sokal \& Rohlf, 1981) with all observations for one sire dropped at a time. A two-way ANOVA on the pseudovalues, treating the estimates obtained by deletion of different sires as belonging to different blocks, tested for differences among nonzero variance components expressed on different hosts. Tukey's Studentized range test was then used to determine which pairs of hosts were different at a 5 per cent significance level (procedure GLM, option TUKEY). The above procedure is equivalent to jackknifing $\ln [($ variance on host 1$) /($ variance on host 2$)]$ for all pairs of hosts, but controls for the experiment-wise rather than comparison-wise type I error. Where the original estimate of a variance component was 0 , all or almost all estimates from reduced data sets were also 0 , and no estimate of standard error was obtained. Because larval survival is an all-or-none trait, variance components could not be estimated as for the metric traits. I estimated heritability of larval survival using the liability-threshold model (Falconer, 1989, chap. 18).

\section{Relationship between variance and host quality and novelty}

To study the relationship between variance components and host quality and novelty one has to standardize the results obtained for different traits for they are measured in different units. Because host species influenced different characters in different ways (T. J. Kawecki, unpublished data), I used a separate indicator of performance for each character rather than a measure of overall host quality. Standardized performance on host $h$ with respect to a given trait $x$ was calculated as $[$ mean $\ln (\mathbf{x})$ on host $h]-[$ mean $\ln (\mathbf{x})$ on all hosts]. Because, in contrast to other traits, an increase in developmental time implied lower performance, I reversed the sign of the performance indicator for that character. The effects of hosts on the variance components were standardized as $\left[\sigma^{2}\right.$ of trait $x$ on host $h$ ]/[average $\sigma^{2}$ of trait $x$ across hosts].
Standardization of quality and variance components permitted pooling the results across traits. I carried out linear regression of standardized variance on standardized performance, and then analysed the residuals in an ANOvA with a planned contrast between mung bean and the three novel hosts. This analysis aimed at detecting the relationship between variance and host quality and novelty. Because growth rate was calculated from emergence weight and developmental time, and fecundity and male life span increased with body size, I repeated this analysis with just four traits: developmental time, emergence weight, residual fecundity and residual life span. It should be noted, however, that the estimates of variance components for different traits were obtained on the same set of individuals, and even the results for these four traits are not quite independent. As a result the $P$ values will be underestimated.

\section{Results}

Heritabilities of posthatching larval survival based on full-sibs were consistently higher than those based on half-sibs, indicating dominance or maternal effects (Table 1). Both heritability estimates were highest on red bean and lowest on mung and adzuki.

The results of variance component analysis for metric traits are summarized in Table 2 . In 10 cases out of the 28 combinations of host and trait, ANOVA detected that the sire component $\sigma_{S}^{2}$, and thus $h^{2}$, were significantly greater than 0 (Table 2 ). In seven out of these 10 cases the estimated dam component $\sigma_{\mathrm{D}}^{2}$ was smaller than $\sigma_{\mathrm{S}}^{2}$ and nonsignificant, although in theory $\sigma_{\mathrm{D}}^{2}$ should be equal to or larger than $\sigma_{\mathrm{S}}^{2}$. The dam component was significant in 17 cases. Because of large standard errors, I detected no significant differences among nonzero estimates of additive genetic variance for any trait, and the dam component differed significantly among hosts only for developmental time. Nevertheless, some patterns do emerge. Mung produced the highest estimates of $\sigma_{\mathrm{S}}^{2}$ for all traits except life span, where it was second highest. Also five out of 10 significant sire component estimates were found on

Table 1 Heritabilities \pm SE of posthatching larval survival in Callosobruchus maculatus

\begin{tabular}{lrc}
\hline Host & \multicolumn{1}{c}{$h_{\text {Half-sibs }}^{2}$} & $h_{\text {Full-sibs }}^{2}$ \\
\hline $\mathrm{Mn}$ & $-0.27 \pm 0.15$ & $0.22 \pm 0.08$ \\
$\mathrm{Ad}$ & $0.07 \pm 0.16$ & $0.14 \pm 0.08$ \\
$\mathrm{Rb}$ & $0.16 \pm 0.17$ & $0.64 \pm 0.11$ \\
$\mathrm{Bg}$ & $0.43 \pm 0.16$ & $0.74 \pm 0.10$ \\
\hline
\end{tabular}

Mn: mung bean; Ad: adzuki; Rb: red bean; Bg: black gram 
mung, including all three cases where the dam component was larger than the sire component, and thus consistent with the genetic interpretation. In turn, adzuki produced the lowest estimates of $\sigma_{\mathrm{S}}^{2}$ for all traits except life span and residual life span. None of the $\sigma_{\mathrm{S}}^{2}$ estimates on adzuki was significantly different from 0 (lowest $P$-value 0.12 ). The dam component $\sigma_{\mathrm{D}}^{2}$ tended to be lowest on red bean.

I found significant differences among hosts in the amount of the within-family variance component $\sigma_{\mathrm{W}}^{2}$ for all characters except male life span and residual male life span. For all five traits exhibiting significant

Table 2 The estimates \pm standard errors of variance components and heritabilities for Callosobruchus maculatus

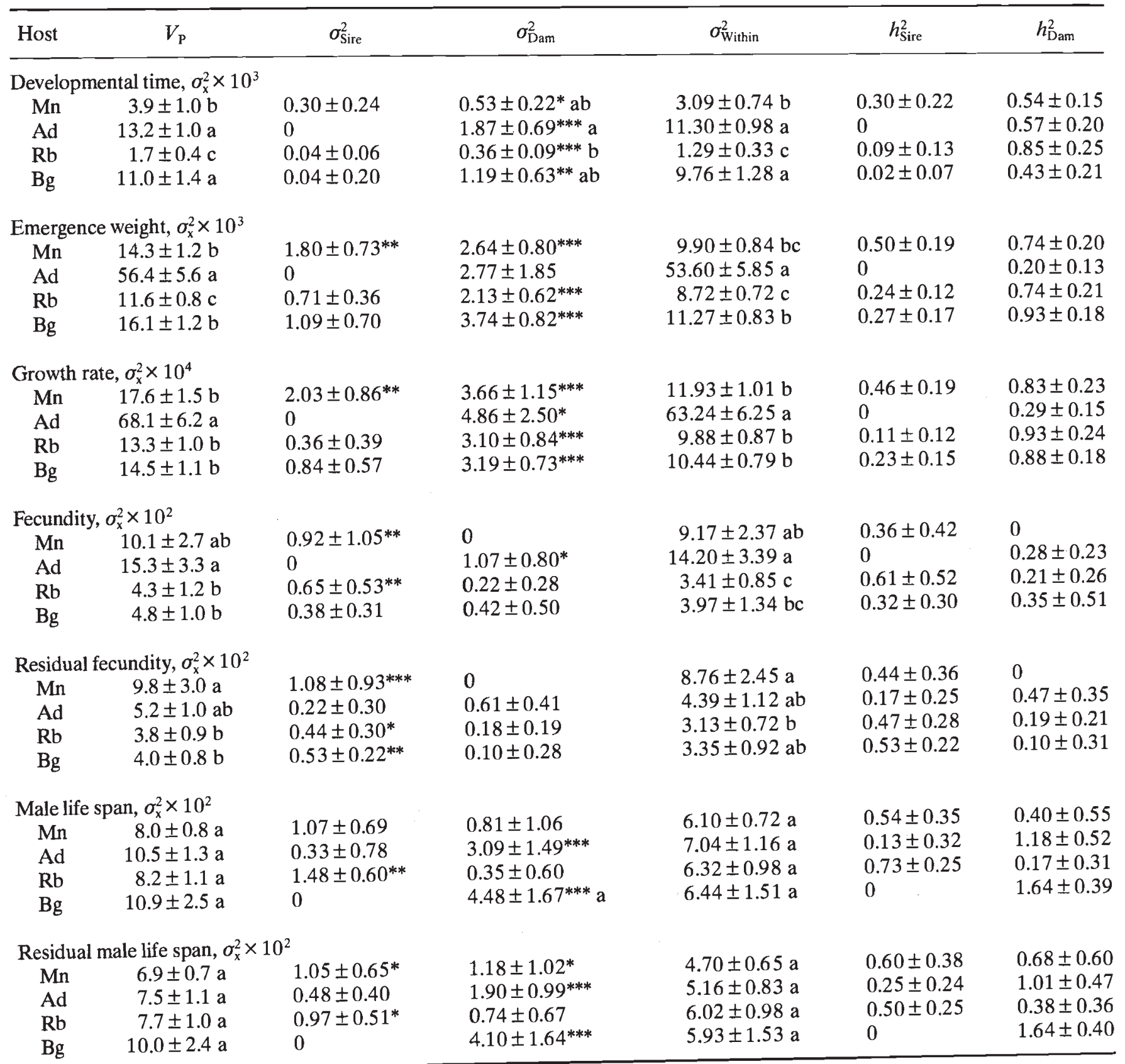

The lower case letters next to the estimates display the results of pairwise a posteriori comparisons (Tukey's test) following an ANOVA on estimates obtained by deleting each sire in turn during the jackknife procedure. Pairs of estimates accompanied by the same letter are not different at the significance level of 0.05 . No differences among nonzero estimates for $\sigma_{\text {Sire }}^{2}$ were detected, and for $\sigma_{\mathrm{Dam}}^{2}$ differences were found only for developmental time. Asterisks denote the significance level of sire and dam components as estimated from an ANOVA parallel to variance component estimation. ${ }^{*} P \leqslant 0.05,{ }^{* *} P \leqslant 0.01,{ }^{* * *} P \leqslant 0.001$ 
differences in $\sigma_{\mathrm{W}}^{2}$ among hosts, the estimates were lowest on red bean; for developmental time $\sigma_{\mathrm{W}}^{2}$ was significantly smaller on red bean than on any other host. For developmental time, emergence weight, growth rate and fecundity the highest within-family variance was expressed on adzuki; for emergence weight and growth rate $\sigma_{\mathrm{W}}^{2}$ was significantly higher on adzuki than on any other host. Because $\sigma_{\mathrm{w}}^{2}$ was by far the largest component of the total phenotypic variance, the pattern of differences in $V_{\mathrm{P}}$ followed that of $\sigma_{\mathrm{W}}^{2}$.

The relationship between standardized performance and the variance components is summarized in Fig. 1. The within-family variance component $\sigma_{\mathrm{W}}^{2}$ for a trait was negatively correlated with the host quality with respect to that character $(N=28$, regression slope $=-2.28, \quad P=0.022$ ). The regression slope remained the same and significant when I included only the four 'independent' characters $(N=16$, slope $=-2.28, P=0.034)$. The sire component tended to increase, and the dam component to decrease, with increasing standardized performance, but neither relationship was significant $\left(N=28 ; \sigma_{\mathrm{S}}^{2}\right.$ : slope $=2.31$, $P=0.09 ; \sigma_{\mathrm{D}}^{2}$ : slope $\left.=-1.80, P=0.12\right)$.

The analysis of variance on residuals from the regression of standardized $\sigma_{\mathrm{s}}^{2}$ estimates on standardized performance revealed that, irrespective of host quality, the beetles expressed consistently more additive genetic variance on mung than on the new hosts (Table 3 ). This difference was highly significant even when only four traits were included in the analysis, and is apparent in Fig. 1 (left panel). I found no significant differences among new hosts. In turn the dam component tended to be smaller on mung than on the novel hosts, although this difference was much less apparent (Fig. 1, middle). The sire and dam components thus showed opposite patterns, and the sum of these two components displayed no relationship with performance or host novelty. For $\sigma_{\mathrm{W}}^{2}$ the points for mung lay close to and on both sides of the regression lines (Fig. 2, right panel). Significant differences existed for both $\sigma_{\mathrm{D}}^{2}$ and $\sigma_{\mathrm{W}}^{2}$ among new hosts. A posteriori tests (Tukey) revealed that they resulted from the estimates on adzuki being higher than on the remaining hosts. The strong tendency for these variance components to be low on red bean disappeared after the estimates were corrected for the effect of high performance on that host. The above results of variance component analysis remained qualitatively unchanged when several outliers with extremely long developmental time were removed from the original data, and when I used variance components estimated from raw rather than log transformed data.

\section{Discussion}

This study shared the common problem of quantitative genetic experiments: large errors of variance component estimates, particularly for fecundity and male life span and their residuals. In addition, because different traits were measured on the same set of individuals, variance component estimates for different characters were not statistically independent, and cannot provide rigorous tests of the hypotheses. Statistical problems notwithstanding, suggestive patterns emerged when the results for different metric traits were standardized and analysed together. The most striking result is that the sire component, which estimates $1 / 4$ of additive genetic variance, was consistently lower on the novel hosts than on mung bean. Although the $P$-values of this trend as given in Table 3 might not be reliable because different traits were measured on the same set of individuals, the pattern is apparent in Fig. 1 (left panel). This result contradicts the prediction of the host novelty hypothesis that more additive genetic variance

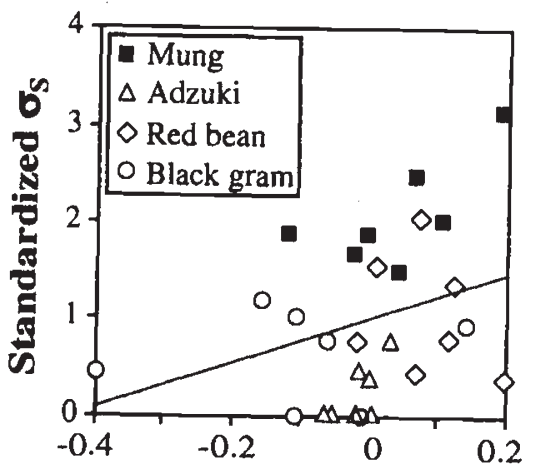

Standardized performance

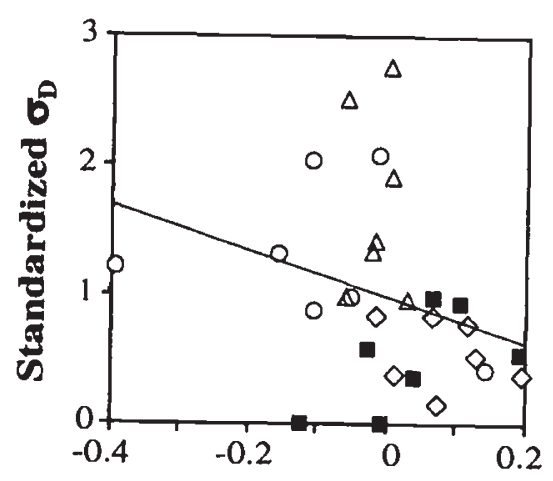

Standardized performance

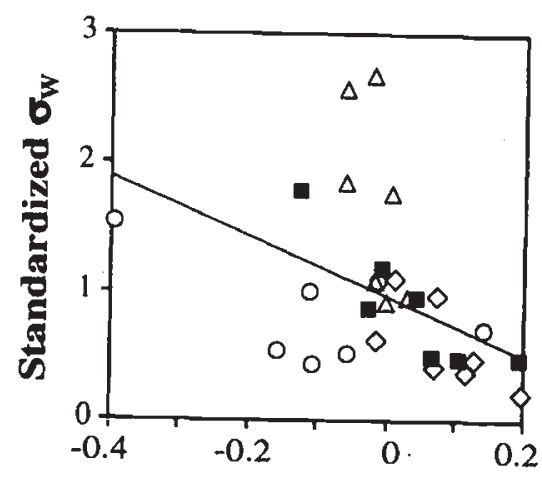

Standardized performance

Fig. 1 Relationship between standardized performance and standardized variance components for seven traits on four hosts. Left panel: the sire component; middle panel: the dam component; right panel: the component within full-sib families. For details of the standardization see Materials and methods. 
Table 3 Analysis of variance of the residuals from the regression of standardized variance components on standardized performance for the seven metric characters of Callosobruchus maculatus

\begin{tabular}{lrlcll}
\hline Source & d.f. & MS & $F$ & $P$ & $P(4$ traits $)$ \\
\hline Sire component $\sigma_{\mathrm{S}}^{2}$ & & & & & \\
Host & 3 & 3.71 & 13.76 & 0.0001 & $(0.0020)$ \\
$\quad$ Mung vs. new & 1 & 9.49 & 35.18 & 0.0001 & $(0.0003)$ \\
$\quad$ Among new & 2 & 0.82 & 3.05 & 0.066 & $(0.34)$ \\
$\quad$ Error & 24 & 0.27 & & & \\
Dam component $\sigma_{\mathrm{D}}^{2}$ & & & & & \\
$\quad$ Host & 3 & 1.72 & 5.61 & 0.0046 & $(0.091)$ \\
$\quad$ Mung vs. new & 1 & 1.87 & 6.12 & 0.021 & $(0.11)$ \\
$\quad$ Among new & 2 & 1.64 & 5.35 & 0.012 & $(0.11)$ \\
Error & 24 & 0.31 & & & \\
Within-family component $\sigma_{\mathrm{W}}^{2}$ & & & \\
Host & 3 & 1.45 & 7.64 & 0.0009 & $(0.10)$ \\
$\quad$ Mung vs. new & 1 & 0.008 & 0.041 & 0.84 & $(0.94)$ \\
$\quad$ Among new & 2 & 2.17 & 11.42 & 0.0003 & $(0.050)$ \\
Error & 24 & 0.19 & & & \\
\hline
\end{tabular}

Numbers in parentheses in the last column are significance levels obtained for the four 'independent traits'

(developmental time, weight, residual fecundity and residual life span).

for performance should be expressed in novel habitats. Taken at face value, it is hard to explain in the light of present evolutionary theories.

The variance component within full sib families, $\sigma_{\mathrm{w}}^{2}$, decreased with increasing performance. This result supports the host quality hypothesis, according to which more environmental variation should be expressed under stressful conditions owing to failure of developmental canalization mechanisms. Apart from environmental variance, $\sigma_{\mathrm{W}}^{2}$ contains parts of additive and dominance variance, but the observed pattern was most likely the result of environmental variance because the genetic components contribute little to $\sigma_{\mathrm{W}}^{2}$, and the additive variance showed an opposite trend. There was no evidence for an effect of host novelty on the environmental variance component.

Estimation of variance components was not possible for larval survival, but the negative heritability estimate on mung suggests very little additive genetic variance for larval survival on mung bean. Heritability of larval survival tended to be higher on red bean and black gram, which were also characterized by higher larval survival than the two other hosts (T. J. Kawecki, unpublished data). This could result from environmental variance being lower on red bean and black gram, in accordance with the host quality hypothesis for environmental variance, or from genetic variance being higher on these hosts, which would contradict the prediction of the host quality hypothesis for genetic variance.

Of course, the results may reflect some specific host properties which are unrelated to their novelty or quality. The differences in environmental variance among hosts may result from different levels of variation in seed quality rather than from effects on developmental stability. To test fully for the effects of host quality and novelty on genetic and environmental variance one would need a broader range of hosts, and several populations that have been maintained on different hosts. Such populations were not available for the South India strain. However, in a recent independent experiment, a different strain ('Brazil') of the cowpea weevil, cultured for many generations on cowpea, expressed less additive genetic variance for female developmental time and emergence weight on mung than on cowpea, and mung was the novel host in that case (John Guntrip, personal communication).

Increased levels of genetic variation are often found in environments characterized by extreme abiotic conditions (reviewed in Hoffmann \& Parsons, 1991). It is not clear, however, whether it is because these conditions are stressful, or because they are novel or rarely encountered. There is some indirect evidence that populations more frequently exposed to stressful conditions express less genetic variation in these conditions than populations that experience them rarely (e.g. Stanley \& Parsons, 1981). The results of this study, in particular the unexpected reduction of additive genetic variance on novel hosts, indicates the need for further research directly addressing the influence of quality vs. novelty of the environment on genetic and environmental variation.

\section{Acknowledgements}

This experiment would not have been possible without extensive help from B. Sykes, O. Kaltz and A. Kawecki. I also thank M. Kaiser, A. Mislin and H. Zingerli for technical assistance, and N. Barton, I. Hanski, V. Perrot, S. Stearns and B. Sykes for their comments on the manuscript. F. Messina provided the experimental animals; C. Dawson, J. Guntrip, R. Sibly and R. Smith offered technical advice. Financial support from the Swiss National Science Foundation (grant no. 3100.028511 .01 to S. C. Stearns), Roche Research Foundation, and Freiwillige Akademische Gesellschaft Basel is gratefully acknowledged.

\section{References}

ARVESEN, J. N. AND SCHMITZ, T. H. 1970. Robust procedures for variance component problems using the jackknife. Biometrics, 26, 677-686. 
FALCONER, D. S. 1989. Introduction to Quantitative Genetics, 3rd edn. Longman, Harlow, UK.

FOX, C. H. 1993. The influence of maternal age and mating frequency on egg size and offspring performance in Callosobruchus maculatus (Coleoptera: Bruchidae). Oecologia, 96, 139-146.

HOFFMANN, A. A. AND PARSONS, P. A. 1991. Evolutionary Genetics and Environmental Stress. Oxford University Press, Oxford, UK.

HOLLOWAY, G. J., POVEY, S. R. AND SIBLY, R. M. 1990. The effect of a new environment on adapted genetic architecture. Heredity, 64, 323-330.

MESSINA, F. J. 1990. Alternative life-histories in Callosobruchus maculatus: environmental and genetic bases. In: Fujii, K. et al. (eds) Bruchids and Legumes: Economics, Ecology and Coevolution, pp. 303-315. Kluwer Academic, Dordrecht, The Netherlands.

MESSINA, F. J. 1991. Life-history variation in a seed beetle: adult egg-laying vs. larval competitive ability. Oecologia, 85, 447-455.

MITCHELL, R. 1991. The traits of a biotype of Callosobruchus maculatus (F.) (Coleoptera: Bruchidae) from South India. J. Stored Prod. Res., 27, 221-224.
ROBERTSON, A. 1955. Selection in animals: synthesis. Cold Spring Harbor Symp. Quant. Biol., 29, 225-229.

SAS INSTITUTE 1989. SAS/STAT User's Guide, version 6. Cary, NC.

SOKAL, R. R. AND RoHLF, F. J. 1981. Biometry, 2nd edn. Freeman, San Francisco, CA.

STANLEY, S. M. AND PARSONS, P. A. 1981. The response of the cosmopolitan species, Drosophila melanogaster, to ecological gradients. Proc. Ecol. Soc. Aust., 11, 121-130.

STEARNS, S. C. AND KAWECKI, T. J. 1995. Fitness sensitivity and the canalization of life history traits. Evolution (in press).

THANTHIANGA, C, AND MITCHELL, R. 1990. The fecundity and oviposition behavior of a South India strain of Callosobruchus maculatus. Entomologia exp. appl., 57, 133-142.

VIA, s. 1991. The genetic structure of host plant adaptation in a spatial patchwork: demographic variability among reciprocally transplanted pea aphid clones. Evolution, 45 , 827-852.

Waddington, C. H. 1940. Organisers and Genes. Cambridge University Press, Cambridge, UK. 